\title{
Study and Evaluate Influence of Minimizing Dimensions HEMT FET Transistor on the (I-V) Curves Characteristics
}

\author{
Omar Ibrahim Alsaif \\ Ph.D. Student. \\ Mosul Technical Institute. \\ Northern Technical Univ. \\ Mosul/ Iraq
}

\author{
Kalid Kaleel Mohammed, $\mathrm{PhD}$ \\ Electronics Engineering College \\ Neniveh Univ. \\ Mosul/ Iraq
}

\begin{abstract}
Continuous miniaturization of HEMTs for the development of ultra high density ICs has resulted in degradation of device parameters by increasing the effects of reducing dimensions. At present time, AlGaN/GaN HEMT is considered as a backbone of both optical and microwave high power electronic applications. Gallium Nitride $(\mathrm{GaN})$ is a wide bandgap semiconductor material with excellent material properties for high frequency, high power, and high temperature electronics. In this work two models of GaN field-effect transistors based on High Electron Mobility Transistors ( HEMT ) technology with different length dimensions ( $4 \mu \mathrm{m}$ and $8 \mu \mathrm{m})$ and channel length ( $0.5 \mu \mathrm{m}, 1 \mu \mathrm{m})$ respectively. Two models have been studied and investigated using Schotcky- Read- Hall ( SRH ) model, which take in account recombination's effects and simulate currents due to voltage field and thermal leakage. Another model ( Fermi- Dirac ) are used as carrier statics model that deals about reducing carrier concentrations in the heavily doped region.
\end{abstract}

Simulation results based on Silvaco Atlas TCAD software for ( I-V) diagrams of the two models, where drain voltage ( 0 to $5 \mathrm{~V})$ and four different gate source voltage applied $(0,-1,-2$, $-3 \mathrm{~V})$. It can be seen the drain current for $(4 \mu \mathrm{m})$ model decreased to $(0.346$ ) when dimensions minimized ( $50 \%$ ), when gate voltage ( $-3 \mathrm{~V})$, while this ratio reduced to ( 0.302 ) at gate voltage ( $0 \mathrm{~V})$, and it will increased as the gate voltage increases, however drain current seems going to saturation region as the drain voltage increased, which gives the best suitability for these types of devices in the radio frequency power applications.

( Ids- Vg ) a good parameter to understand effects of reducing device dimensions, where the current ranging $(20-70 \mu \mathrm{A})$ for $(4 \mu \mathrm{m})$ and $(1.01-1.09 \mathrm{~mA})$ for the other model, at the same voltage conditions. The work has contributed to a substantial improvement in the area of device simulation and increased efficiency in device design in general, but particularly for ( GaN) HEMT nanometric technologies.

\section{Keywords}

HEMT, GaN properties, SRH model, dimensions effect, Silvaco Atlas TCAD software, ( I-V ) characteristics.

\section{INTRODUCTION}

Three types of crystal structures exist for majority of group III-nitride devices: Wurtzite (hexagonal), zinc-blende and rock salt structures. Thermodynamically stable phase under ambient conditions is the Wurtzite structure where as a metastable type of structure is zinc-blende which is formed when a film is grown on a cubic substrate like GaAs and $3 \mathrm{C}$ $\mathrm{SiC}$. The rock salt structure does not exist naturally and can only be developed under high pressure condition. The
Wurtzite structure has a hexagonal unit cell with two lattice constants, ( a ) and ( c ) as shown in the figure below [ 1].

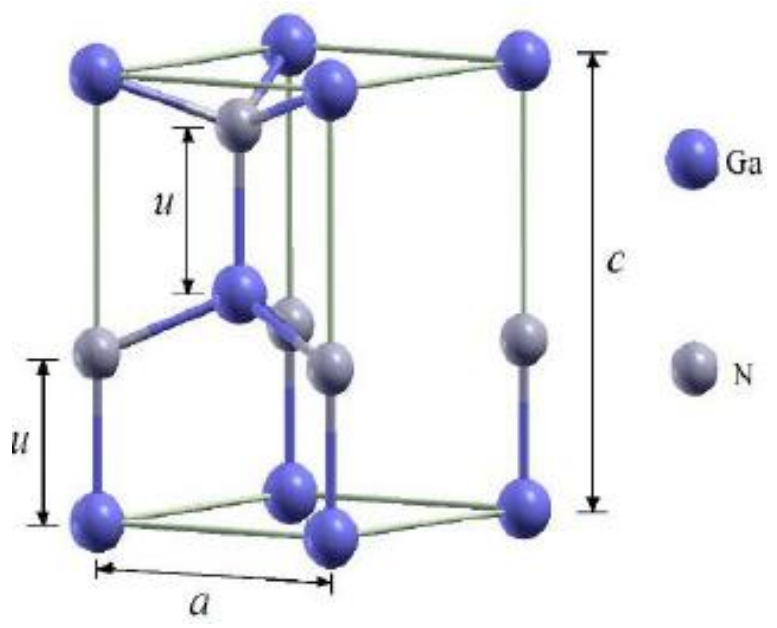

Fig. 1: The Wurtzite structure of GaN.

On the other hand, zinc-blende structure has a cubic unit cell and possesses higher degree of crystallographic symmetry because of having equal lattice constants in three perpendicular directions [2]. GaN devices usually exist in Wurtzite structure which can be grown with two polarities (Ga-face and $\mathrm{N}$-face) along the $\mathrm{c}$ axis subject to the atomic order through growth direction. The presence of strong spontaneous and piezoelectric polarization effects is a unique feature of Wurtzite group III-nitride structures [3][4].

\subsection{Electrical Properties}

A semiconductor material should possess the following properties: large energy band gap, high thermal conductivity, low dielectric constant and large breakdown field for high frequency and high power applications. Large band gap enables with standing high internal electric field before the occurrence of breakdown and provides enhanced radiation resistance. The dielectric constant of large band-gap semiconductors is nearly $20 \%$ lower than the other conventional semiconductors which allow them to be about $20 \%$ larger in the area for a given impedance. Increased an area leads to higher RF currents and higher RF power thereby. Significant material properties of $\mathrm{GaN}$ in comparison with other semiconductor materials are shown in Table(1) [5]-[6].

Result from GaN HEMT based circuits, as a result, smaller capacitors and inductors are needed which minimize overall size and cost. 
Table 1: Material properties of various semiconductors

\begin{tabular}{|c|c|c|c|c|c|}
\hline Property & $\mathrm{Si}$ & GaAs & $4 \mathrm{H}-\mathrm{SiC}$ & $6 \mathrm{H}-\mathrm{SiC}$ & $\mathrm{GaN}$ \\
\hline Bandgap (eV) & 1.12 & 1.42 & 3.27 & 3.02 & 3.4 \\
\hline $\begin{array}{l}\text { Breakdown electric field } \\
\text { (MV/cm) }\end{array}$ & 0.3 & 0.4 & 3 & 3.2 & 3.3 \\
\hline Relative dielectric constant & 11.7 & 12.9 & 9.7 & 9.66 & 8.9 \\
\hline $\begin{array}{c}\text { Thermal conductivity } \\
(\mathrm{W} / \mathrm{mK})\end{array}$ & 150 & 55 & 370 & 490 & 130 \\
\hline Electron mobility $\left(\mathrm{cm}^{2} / \mathrm{V} . \mathrm{s}\right)$ & 1400 & 8500 & 900 & 400 & 1000 \\
\hline $\begin{array}{l}\text { Saturated electron drift } \\
\text { velocity } \\
\left(\times 10^{7} \mathrm{~cm} / \mathrm{s}\right)\end{array}$ & 1 & 1 & 2 & 2 & 2.5 \\
\hline Melting point $(\mathrm{K})$ & 1415 & 1238 & 2827 & & 2791 \\
\hline Hole mobility $\left(\mathrm{cm}^{2} / \mathrm{Vs}\right)$ & 450 & 400 & 120 & 90 & 200 \\
\hline
\end{tabular}

\section{HEMT STRUCTURE}

A hetero structure is usually formed by joining two different materials with different bandgaps HEMT, the structure consists of a thin and strained layer of $\mathrm{AlGaN}$ on top of a thick relaxed GaN layer. The heterointerface is formed at the junction of these two layers acting as two dimensional electron gas (2DEG) channel. $\mathrm{AlGaN}$ and $\mathrm{GaN}$ layers are known as barrier and buffer layers respectively. The metal contacts used for source and drain terminals are Ohmic contact whereas Schottky contact is used at the gate end. $\mathrm{AlGaN}$ can be moderately doped or undoped or unintentionally doped. GaN layer is left undoped [7].

A typical cross-sectional schematic of AlGaN/GaN HEMT device is shown in Figure(2). The device is usually grown on a semi-insulating substrate which has a high thermal stability and close lattice matching with $\mathrm{GaN}$. A buffer layer is grown on top of the substrate to act as an isolation layer between the substrate and channel. Any lattice mismatching or crystal defects from the substrate are minimised using this $\mathrm{GaN}$ buffer layer.

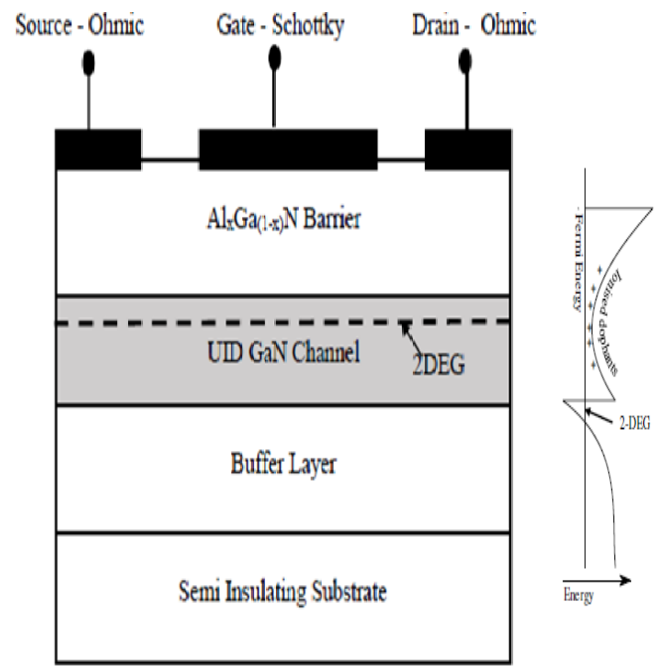

Fig. 2: Layer structure of a typical AlxGa (1-x)N/GaN HEMT.

\section{HEMT OPERATION}

In the most common HEMT structures, the wide band gap barrier is doped n-type while the narrow bandgap channel remains undoped. As a result, electrons diffuse from the wide bandgap material into the narrow bandgap material to minimize their energy. This process continues until a balanced Fermi level is formed in the two materials and equilibrium is established as shown in figure (2). Because of the resulting electrostatics, a new triangular well forms on the narrow bandgap side of the heterojunction. Which is called as two dimensional quantum well and the electrons confined inside the well is called Two Dimensional Electron Gas (2DEG).

The $n$-doped barrier in the device supplies electrons to the undoped channel, thus spatially operating the channel charge carriers from their ionized donors. In this manner, the heterostructure channel is capable of delivering high carrier concentration with high mobility as impurity scattering is minimized in the undoped channel. As an added advantage, surface scattering is also reduced by moving the currentcarrying region below the barrier [8].

\section{SILVACO: TCAD SIMULATION SUITE}

Device simulation is extensively used to progress and optimize the semiconductor procedures to design and predict the electrical characteristics of the device under test. A device structure is simulated by defining the physical structure; it includes material specification, device models, numerical methods, etc. These models and measured parameters help in solving the physical equations at identified bias environments. Atlas simulations execute the command and structure files to generate the corresponding output files which store the generated terminal voltages and currents obtained from the device analysis and also the solution files containing 2D and $3 \mathrm{D}$ solution data variables within the device at a given bias are stored and plotted using tools like Tony plot .

Silvaco's ATLAS is a versatile and modular program designed for one, two, and three-dimensional device simulation. BLAZE and GIGA, are ATLASsub-modules structure shown in (Figure 3) which perform specialized functions required for advanced materials, heterojunctions, and temperature-dependent conditions [11].

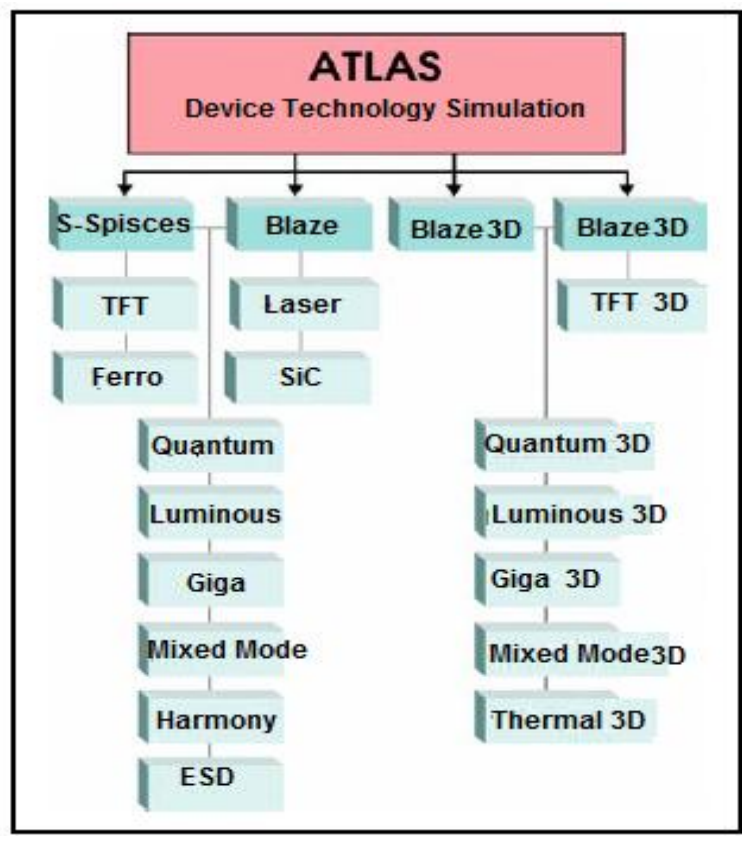

Fig.3: Representation of ATLAS' modular structure.

There are several steps are essential to make a transistor model based on ( Fermi- Dirac) and Schockley Read Hall ( SRH model ) by silvaco software. Determine regions number, which Meaning different material types and its deposition boundaries as illustrated in the table (2). 
Table 2: Deposition boundaries for each needed material in Silvaco software.

\begin{tabular}{|c|c|c|c|}
\hline $\begin{array}{c}\text { Region } \\
\text { No. }\end{array}$ & $\begin{array}{c}\text { Material } \\
\text { Type }\end{array}$ & Ymin(Micron) & Ymax(Micron) \\
\hline 1 & $\mathrm{SiO}_{2}$ & 0 & 0 \\
\hline 2 & $\mathrm{GaN}$ & 0 & 0.005 \\
\hline 3 & $\mathrm{AlN}$ & 0 & 0.1 \\
\hline 4 & $\mathrm{GaN}$ & 0.01 & 1.0 \\
\hline
\end{tabular}

The exact structure dimensions shown in Figure (4) and figure (5) selected for this paper is Contains detailed information for the component materials for the two types of model

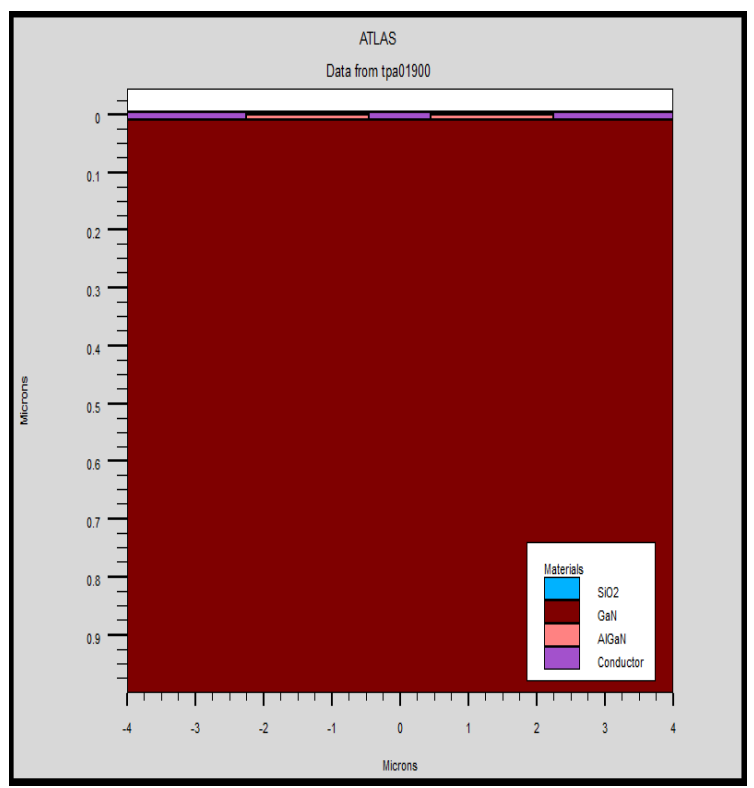

Fig. 4: model structure ( $8 \mu \mathrm{m}$ ).

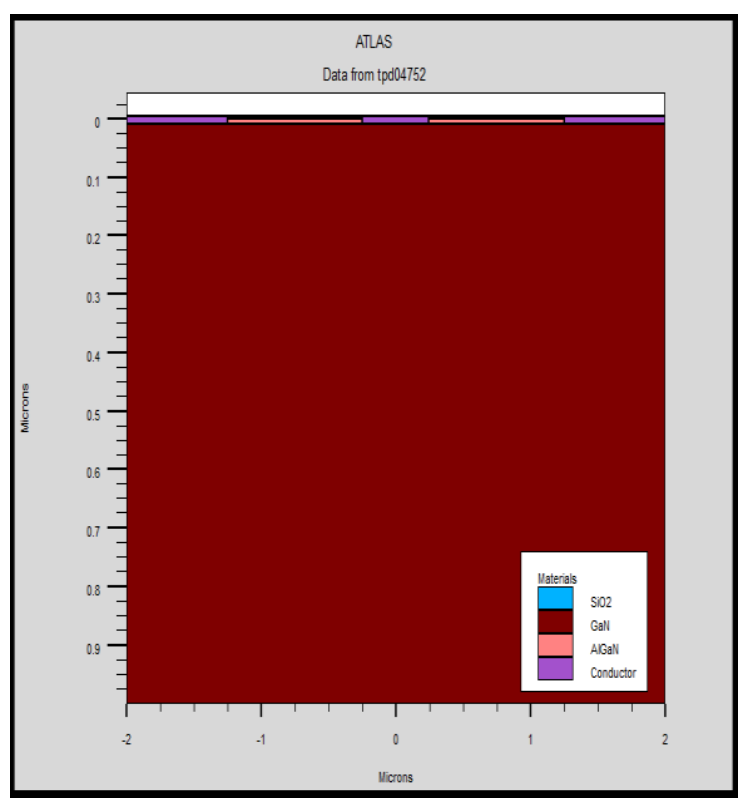

Fig. 5: model structure ( $4 \mu \mathrm{m})$.
It is very clear that all the dimensions of the second model are twice the dimensions of the first one, as shown in table (3).

Table 3: explain the dimension of designed models

\begin{tabular}{|c|c|c|}
\hline & $\begin{array}{c}8(\mu \mathrm{m}) \\
\text { model }\end{array}$ & $4(\mu \mathrm{m})$ Model \\
\hline $\mathrm{Lg}$ & 1.0 & 0.5 \\
\hline $\mathrm{Ld}=\mathrm{Ls}$ & 2 & 1.0 \\
\hline $\begin{array}{c}\mathrm{Lgd}= \\
\mathrm{Lgs}\end{array}$ & 1.5 & 0.75 \\
\hline
\end{tabular}

\section{5. (CURRENT - VOLTAGE)} CHARACTERISTICS

The linear region of the channel under gate contains drainsource current which affected mainly by source and drain resistances expressed as[9]:

$\int_{0}^{L} I_{d s} d x=q Z \mu \int_{I_{d s} R s}^{V d s-I d s R d} N d(a-h) d V .$.

$I_{d s}=\frac{q Z \mu N d a}{L}\left(\left(V d s-I d s(R s+R d)-\frac{2}{3 V_{P}^{1 / 2}}[(V b i-\right.\right.$

$\left.\left.\left.\left.V g s+V d s-I_{d s} R_{d}\right)^{3 l 2}\right]\right)\right) . .($

Where $I_{d s}$ is the drain-source current, $\mu$ the electron mobility, a, the active channel thickness, $\mathrm{L}$ and $\mathrm{Z}$, the gate length and width respectively, Vds, the applied drain-source voltage, Rs and $\mathrm{Rd}$ the parasitic source and drain resistances, and $\mathrm{Vp}$, the pinch off voltage [9]. Silvaco software solve the above equations to get the value of current, according to the considered values of ( Rs and Rd ). At higher drain-source voltage, the electric field in the conducting channel increased which leads to velocity saturation. There is substantial extension of the depletion region beyond the gate forming high field region at the drain side of the channel.

To model this high field region, silvaco software consider the gate length modulation effect and the potential in this region calculated from ( 2-D Laplas equation ).

2-D potential $((x, y)$ in the high region, keeping only the first term of the series as shown below[10]:

$\psi(x, y)=\frac{2 a}{\pi} \operatorname{Es} \operatorname{Cos}\left(\frac{\pi y}{2 a}\right) \operatorname{Sinh}\left(\frac{\pi(x-L)}{2 a}\right)$

Where $E s=\frac{V_{\text {sat }}}{\mu}$ is the velocity saturation field, $V_{\text {sat }}$. as the saturation velocity

\section{RESULTS ANALYSIS AND DISCUSSION}

Simulation results based on silvaco software for (I-V) diagrams of the two models, where drain voltage ( 0 to $5 \mathrm{~V}$ ) and four different gate source voltage applied $(0,-1,-2,-3$ V), were shown in Figure.(6) and (7). 


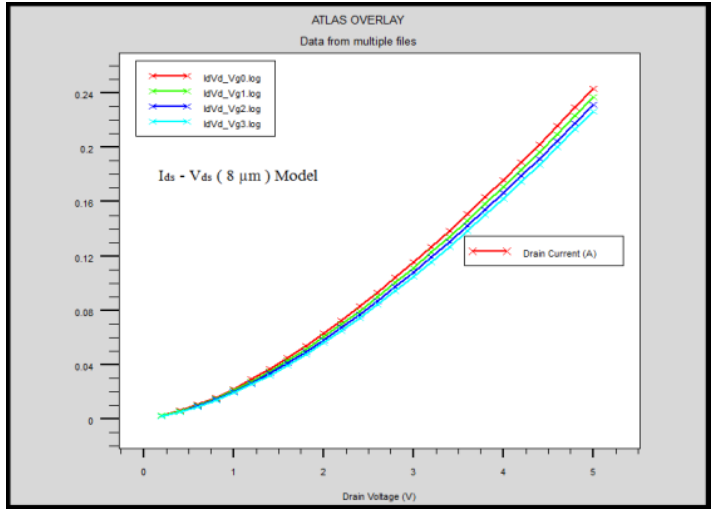

Fig.6 : illustrate ( I-V ) curves for ( $8 \mu$ )model

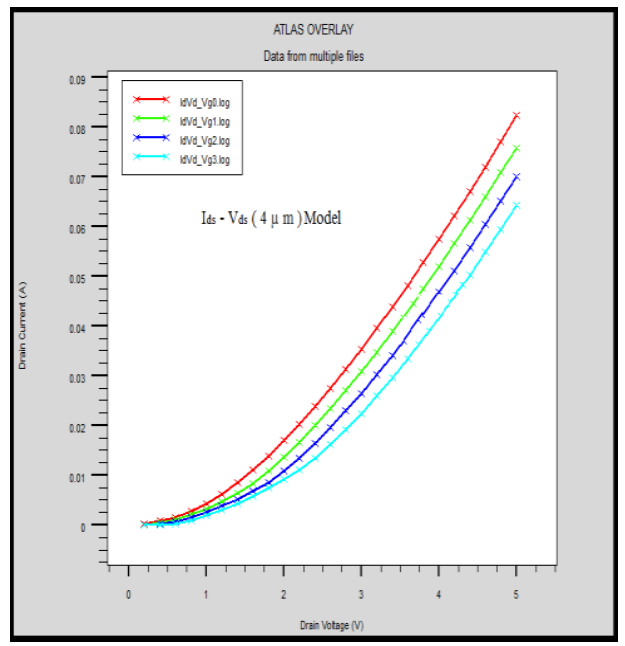

Fig.7: illustrate ( I-V ) curves for $(4 \mu)$ model

It can be seen that from the above figures that the drain current for $(8 \mu \mathrm{m})$ has a value $(0.08 \mathrm{~A})$ at $\quad(1.0 \mathrm{~V})$, while other model has ( $0.01 \mathrm{~A})$ at same voltage drain and gate. This value increased to its maximum ( $0.24 \mathrm{~A})$ at voltage drain $(5 \mathrm{~V})$ for $\quad(8 \mu \mathrm{m})$ model, while it is $(0.08$ A ) at same voltage for other model. These changing in curve characteristics as a result of minimizing dimensions are reflected to the main important region of the short channel effect.

Another good parameter which indicates the powerful of the device performance is ( Ids - Vgs ). Figure ( 8 ) and (9) illustrates curve characteristics for drain current against gate voltage for each models.

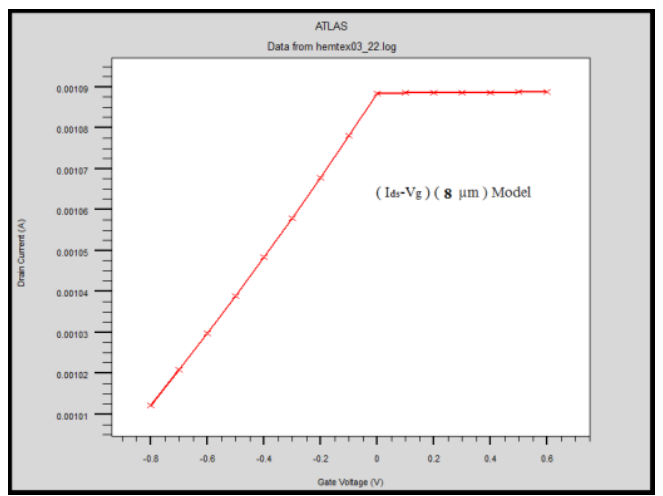

Fig. 8: ( Ids - Vg ) curve for $(8 \mu)$ model



Fig. 9: ( Ids - Vg ) curve for $(4 \mu)$ model

It is clear that the curves have the same shape and arcs, but they differ in terms of values, and this is consider as a result of minimizing the dimensions of the second model and its influence that extend to most of the device characteristics for their components, where they affect to the all values of the capacitance and frequencies.

\section{CONCLUSION}

In this paper, an attempt is made to study and evaluate the influence of minimizing dimension of two HEMT transistors models based on famous materials $\mathrm{GaN}$ with typical dimensions ( $8 \times 1 \mu \mathrm{m})$ and ( $4 \times 1 \mu \mathrm{m})$ with channel length ( $1.0 \mu \mathrm{m}$ and $0.5 \mu \mathrm{m})$ respectively. For (I-V) characteristics, ( $8 \mathrm{x} 1 \mu \mathrm{m})$ model shows large current drain according to drain voltage. This high current and output power makes this model very suitable for power amplifier applications, but on the expense of device area and size. It can be conclude the drain current for $(4 \mu \mathrm{m})$ model decreased to $(0.346)$ when dimensions minimized ( $50 \%)$, at gate voltage ( $-3 \mathrm{~V})$, while this ratio reduced to $(0.302)$ at gate voltage ( $0 \mathrm{~V})$. A methodological matching of two model GaN-based HEMTs with different dimensions to calculate ( current- voltage ) characteristic which physically based on Silvaco-"ATLAS" simulation program was developed, where it was found that reducing the dimensions by half will lead to decrease ( I-V ) curve to about one-third.

\section{REFERENCES}

[1] del Alamo, J. A., and Joh, J. (2009). GaN HEMT reliability. Microelectronics reliability, 49(9), 12001206.

[2] Sumiya, M., and Fuke, S. (2004). Review of polarity determination and control of GaN. MRS Internet Journal of Nitride Semiconductor Research, 9, e1.

[3] Rivera, C., and Munoz, E. (2009). The role of electric field-induced strain in the degradation mechanism of AlGaN/GaN high-electron-mobility transistors. Applied Physics Letters, 94(5), 053501.

[4] Joh, J., and Del Alamo, J. A. (2008). Critical voltage for electrical degradation of $\mathrm{GaN}$ high-electron mobility transistors. IEEE Electron Device Letters, 29(4), 287289.

[5] Foutz, B. E., O’Leary, S. K., Shur, M. S., and Eastman, L. F. (1999). Transient electron transport in wurtzite GaN, InN, and AlN. Journal of Applied Physics, 85(11), 77277734. 
[6] Tolbert, L. M., Ozpineci, B., Islam, S. K., and Chinthavali, M. S. (2003). Wide bandgap semiconductors for utility applications. Semiconductors, 1,3 .

[7] Faqir, M., Verzellesi, G., Chini, A., Fantini, F., Danesin, F., Meneghesso, G., Zanoni, E. and Dua, C. (2008). Mechanisms of RF current collapse in $\mathrm{AlGaN}-\mathrm{GaN}$ high electron mobility transistors. IEEE Trans. Device and Materials Reliability, 8(2), 240-247.

[8] Morkoc, H. (2008). Handbook of Nitride Semiconductors and Devices, 1. Wiley-vch.
[9]. Manju K. Chattopadhyay, Snjiv Tokikar "Thermal model for dc characteristics of algan/gan hemts including selfheating effect and non-linear polarization" Microelectronics Journal Volume 39 Issue 10, October, Pages 1181-1188, 2008.

[10] Joachim Piprek (2007) Nitride Semiconductor Devices Principles and Simulation WILEY-VCH Verlag GmbH \& Co. KGaA, Weinheim

[11] "ATLAS User's Manual, Device Simulation Software", 2010 ed Santa Clara, CA.: SILVACO International, 2010 . 